\title{
Barriers and Facilitators to Implementing a Stepped Care Cognitive-behavioral Therapy for Insomnia in Cancer Patients: a Qualitative Study.
}

Josée Savard ( $\sim$ josee.savard@psy.ulaval.ca )

Université Laval: Universite Laval https://orcid.org/0000-0002-8052-1046

Catherine Filion

CHU de Québec-Université Laval Research Center

Marie-Pierre Gagnon

Universite Laval Faculte des Sciences Infirmieres

Aude Caplette-Gingras

Université Laval Cancer Research Center

Lynda Bélanger

Brain Research Center: Centre de recherche CERVO

Charles M. Morin

Université Laval: Universite Laval

Research

Keywords: insomnia, cancer, cognitive-behavioral therapy, stepped care, self-administered treatment, webbased intervention, implementation, facilitators, barriers

Posted Date: January 18th, 2021

DOl: https://doi.org/10.21203/rs.3.rs-147791/v1

License: (9) This work is licensed under a Creative Commons Attribution 4.0 International License.

Read Full License 


\section{Abstract}

Background: Insomnia affects between 30 to $60 \%$ of cancer patients and tends to become chronic when left untreated. Cognitive-behavioral therapy for insomnia (CBT-I) is the recommended first-line treatment for cancer-related insomnia. Yet, this treatment is not readily accessible. A stepped care intervention beginning with a self-administered (web-based) intervention appears to be a promising cost-effective approach to offer CBT-I in routine cancer care as compared to a standard face-to-face therapy. This qualitative study investigated current practices in the assessment and management of insomnia in five hospitals offering cancer care and identified the barriers and facilitators to the implementation of a stepped care CBT-I in these clinical settings.

Methods: Nine focus groups composed of a total of 43 clinicians (e.g., physicians, nurses, nurse navigators, technologists, psychologists) and administrators, as well as 10 cancer patients were held. The Consolidated Framework for Implementing Research was used to develop the semi-structured interview and analyze the data.

Results: Sleep difficulties are not systematically discussed in clinical practice and when a treatment is offered, most often, it is a pharmacological one. Based on the Consolidated Framework for Implementing Research, barriers to the implementation of a stepped care CBT-I included individual characteristics (e.g. lack of knowledge about CBT-I among cancer care providers, patients' comorbidities and preferences); intervention characteristics (e.g., lack of internet access); inner setting characteristics (e.g., lack of time and resources, resistance to change); and process factors (e.g., need for prior training and engagement of all stakeholders). Facilitators were related to individual characteristics (e.g. strong beliefs in the efficacy of CBT-I); intervention characteristics (e.g. web-based format that increases accessibility at a lower cost, short- and long-term effects of CBT-I); and process factors (e.g. high motivation and commitment to offer a new service to patients).

Conclusions: This qualitative study confirms the need to better address insomnia in routine cancer care and suggests that, while some barriers were mentioned, the implementation of a stepped care CBT-I is feasible provided that some conditions are met such as prior training and engagement of all stakeholders from the outset.

\section{Contributions To The Literature}

- This study is the first to assess factors that could facilitate or impede the integration of a nonpharmacological treatment for insomnia in routine cancer care.

- The treatment (i.e., cognitive-behavioral therapy) used is the recommended first-line treatment for chronic insomnia, yet it is not widely accessible.

- Cognitive-behavioral therapy for insomnia is offered within a stepped care approach beginning with a self-administered (web-based) intervention followed, if needed, by up to three sessions with a clinician. 
- This treatment delivery is innovative and more easily implementable in routine care. As such, it is likely to considerably improve access to this evidence-based intervention when implemented.

\section{Background}

Between $30 \%$ and $60 \%$ of cancer patients report insomnia at some point during their cancer care trajectory (1). Insomnia tends to become chronic when not adequately treated, which is the rule rather than the exception (1). Untreated insomnia may lead to several negative consequences (e.g., development of depressive disorders) and costs related to chronic insomnia for the patient and society (2) largely outweigh those of an effective treatment for this problem (3). Cognitive-behavioral therapy for insomnia (CBT-I) is the treatment of choice for chronic insomnia (4),(5) and has been recommended as the first line treatment for cancer-related insomnia (6). In fact, CBT-I has been found to be efficacious in cancer patients when administered in a standard format (i.e. face-to-face sessions with a therapist; (7),(8),(9). However, given the time required, the associated costs, and the lack of qualified practitioners, access to CBT-I is very limited in oncology clinics (10),(11),(12).

Various forms of self-administered CBT-I have been developed to make this treatment more accessible (e.g., bibliotherapy, web-based). The efficacy of self-administered CBT-I is well established for insomnia unrelated to a medical condition (13),(14),(15). Compared to a face-to-face intervention, self-administered CBT-I is more readily accessible, can be provided at a lower cost, allows more flexibility to patients and requires lower treatment intensity and less specialist time (14). However, the authors of a meta-analysis concluded that self-administered CBT-I led to smaller sleep improvements than standard CBT-I (13). This is consistent with results of our previous randomized controlled trial (RCT) in women with breast cancer which showed that a video-based CBT-I (videos and booklets) produced significantly greater sleep improvements than a no-treatment control group, but significantly smaller effects than a standard faceto-face CBT-I on some key variables, including insomnia severity (16). However, the video-based CBT-I was much more cost-effective (17).

Nonetheless, we concluded that self-administered CBT-I would better be used as part of a stepped care model. Indeed, there is a high probability of under treatment if patients do not receive more intensive therapies when sleep improvement is still below the normative range after receiving a self-administered treatment (14). Stepped care models have been proposed as a cost-effective way to make CBT-I more widely accessible (18),(19). Typically, stepped care models of care begin with a low intensity treatment, such as a self-administered intervention, which is followed, if the patient is not remitted, by a more intensive form of intervention. Stepped care treatments are less demanding in terms of professional resources and are, thus, less costly, which makes their implementation in routine care more likely (18), (20). We conducted the first RCT comparing the efficacy of a stepped care CBT-I to that of a standard professionally-administered treatment (17). Stepped care patients who had less severe insomnia first received a web-based intervention followed, if they were still symptomatic, by up to three face-to-face sessions with a clinician. The trial used a non-inferiority design and was conducted in patients with 
mixed cancer sites $(N=177)$. Results showed that stepped care CBT-I was significantly non-inferior to standard CBT-I (6 face-to-face sessions) in reducing insomnia severity and improving sleep efficiency.

Although stepped care CBT-I appears to be an efficacious alternative to standard CBT-I, it is now important to assess how feasible its implementation is in routine cancer care. The first step consists of identifying barriers and facilitators to implementation among the various stakeholders (21). This qualitative study aims at characterizing the current practices in the assessment and management of cancer-related insomnia and at identifying the main factors that could facilitate or hinder the implementation of a stepped-care CBT-I using the Consolidated Framework for Implementing Research (CFIR) (22). The CFIR considers a broad range of factors at the innovation, individual, contextual and process levels, and it proposes a pragmatic approach to make changes in complex environments such as healthcare organizations.

\section{Methods}

\section{Setting and Participants}

The study was conducted in five hospitals (L'Hôtel-Dieu de Québec [L'HDQ], Hôpital du St-Sacrement [HSS], Hôpital de l'Enfant-Jésus [HEJ], all part of the CHU de Québec-Université Laval, Hôtel-Dieu de Lévis [HDL] and CISSS Chaudière Appalaches (CISSSCA) and Institut universitaire de cardiologie et de pneumologie de Québec [IUCPQ]) providing cancer care in the Quebec city metropolitan area (Quebec, Canada). The sample comprised clinicians (nurses, psychologists, social workers, pharmacists, and physicians) and hospital administrators $(n=49)$, as well as patients who received cancer treatment and experienced sleep difficulties $(n=10)$.

Clinicians and administrators were identified by participating clinics' coordinators of psychosocial oncology services who had to make sure that at least one member of each type of professional involved in the screening and management of psychosocial disturbances in their hospital was represented. Potential participants were contacted by email to explain the study goals and to determine their interest in taking part in it. The study was approved by the ethics' committee of each institution (CHU de QuébecUniversité Laval: \# MP-20-2020-4796; HDL: \# MEO-20-2020-711; IUCPQ : \#MEO-20-2021-3408).

The first group of patients had already completed the program as part of our prior RCT ((17); $n=8)$ and were recruited by phone from among participants who had agreed to be contacted for another study. The other patients, recruited in the surgical oncology clinic waiting room of $\mathrm{CHU}$ de Québec-Université Laval, did not complete the insomnia treatment $(n=2)$. A telephone interview was scheduled to verify patients' eligibility for the study. The inclusion criteria were: 1 ) to be healthy; 2 ) to have a sufficient level of health literacy;3) to have antecedents or current insomnia; 4) to have a sufficient level of French; and 5) to be able to travel to attend the focus group. Exclusion criteria were: 1) metastatic cancer; and 2) severe cognitive impairment or psychological disorder. 
All participants were invited to take part in a focus group about the acceptability and feasibility of implementing a new intervention addressing sleep difficulties in routine cancer care. The date and time of the focus group were sent by email, along with the consent form and a socio-demographic questionnaire. A professional facilitator, from the research firm SOM, conducted the discussions. No compensation was

offered for the clinicians and administrators of the CHU de Québec-Université Laval, HDL and CISSSCA as focus groups were held during regular business hours in the participants' workplace. The IUCPQ administration requested that the research team defray the costs related to the participation of nurses at their usual hourly rate. To compensate for the time invested and their travel expenses, patients received $\$ 75.00$.

\section{Material and Methods}

Participants first had to read and sign the consent form and to complete a sociodemographic questionnaire. Before the discussion began, a brief introduction of cancer-related insomnia (e.g., prevalence) and CBT-I (e.g., efficacy) was presented and our stepped care CBT-I was described; i.e., webbased CBT-I (www.insomnet.com) for all patients followed if needed, for patients still symptomatic, by up to three booster sessions with a clinician. Two distinct semi-structured interviews were used for hospital employees and patients. The interview guides with a set of predetermined questions were developed based on the CFIR and the systematic review of staff-reported barriers and facilitators to the implementation of hospital-based, patient-focused interventions by Geerligs et al. (23). The employee version covered their current practice with cancer patients having sleep problems, their knowledge and beliefs about CBT-I, their potential interest in a stepped care CBT-I, their perceived barriers and facilitators to its implementation in their own clinical setting, and their suggestions for the implementation process. For patients, the themes discussed were: current presence of sleep problems, their opinion on current practices of assessing and treating cancer-related insomnia, their potential interest (and satisfaction for those who received it) in CBT-I and in a stepped care model to make it more widely accessible, and their perceived barriers and facilitators to implementing it in routine cancer care. The duration of interviews ranged from 65 to 90 minutes.

\section{Data analysis}

Focus groups were audio recorded and transcribed verbatim by two undergraduate psychology students. Verbatim transcriptions were imported in NVivo 11.0 (24), and a hybrid inductive-deductive thematic analysis was performed (25). The first two focus groups conducted with employees were analyzed independently by two raters (MPG and one research professional) with significant experience in qualitative analyses who compared their coding and came to consensus on a common codification tree. Then, they met with JS and CF who proposed some additional codes that were added to the codification tree. The seven remaining focus groups were analyzed by the same research professional and then validated by MPG, JS and CF. 


\section{Results}

\section{Composition of Focus Groups and Participants' Demographic Characteristics}

A total of 43 clinicians (nurse navigators [ $n=10]$, nurses [ $n=8]$, psychologists [ $n=10]$, social workers [ $n=$ 6], pharmacists [ $n=2]$, physicians $[n=4]$, and radiation oncology technologists $[n=3]$ ) and 6 hospital administrators participated in one of the nine focus groups conducted with hospital employees (Table 1). Two groups were conducted at L'HDQ because of the high number of professionals involved. Additionally, 10 patients who received cancer treatments and experienced sleep difficulties participated in two focus groups. Among these, eight patients had received the stepped care CBT-I as part of our previous RCT (17).

The majority of clinicians and administrators were women $(n=45 ; 91.8 \%)$, were married or in a committed relationship ( $n=36 ; 73.5 \%)$ and had completed a university degree $(n=22 ; 44.9 \%)$. They were on average 41 years old (range: $28-61$ ). Patients were on average 60 years old (range: $47-74)$ and a majority was female ( $n=9 ; 90 \%)$, was married or in a committed relationship $(n=4 ; 40 \%)$ and had completed a university degree $(n=7 ; 70 \%)$. The cancer sites were: breast $(n=6 ; 60 \%)$; prostate $(n=1$; $10 \%)$; gynecological $(n=1 ; 10 \%)$; central nervous system $(n=1 ; 10 \%)$; and salivary gland $(n=1 ; 10 \%)$.

\section{Current Practices in the Assessment and Treatment of Cancer-Related Insomnia}

Discussions about sleep. Patients were unanimous in saying that cancer care providers did not enquire about the quality of their sleep. Yet, this is a significant problem.

I'd never heard that there could be a link between cancer and insomnia. I'd had a little [insomnia] before, so I took a few pills from time to time (Patient).

Assessment of sleep problems by cancer care providers. When sleep problems are reported by their patients, first-line providers try to assess the possible reasons for these difficulties and to recommend basic management strategies based on that information.

Personally, I do a quick assessment to find out: is it recent? Has it gotten worse since the illness was diagnosed? Do they know about relaxation techniques?... What do they do when it occurs? Do they take medication? Then [I teach] relaxation techniques and all of that (Social Worker).

What I do at the beginning is I try to see why they have insomnia...So, at the beginning I try giving the good old advice, take naps in the afternoon but not too long, stay away from caffeine, chocolate in the evening, exercise, yes they can rest but that doesn't just mean staying in bed (Nurse).

Patients stated that sleep should be assessed and discussed right after the cancer diagnosis. 
There should be a follow-up right away because the worst time is between the diagnosis and the first treatments. At the beginning, right after the diagnosis. At least make them [patients] aware that it can occur, that they might have trouble sleeping (Patient).

Current management of sleep difficulties. Both patients and hospital professionals mentioned that when insomnia treatment is initiated, it is most frequently of a pharmacological nature. Hypnotics are seen as more appropriate in some acute situations (e.g., when receiving Decadron, absence of comorbidity) while the limitations of a chronic hypnotic use (e.g., risk of dependence) are well recognized.

When I spoke to my doctor about it, at the beginning, he offered me pills to relax me (Patient).

The nurses work hard and they're kind, but there comes a time, after they've done two shifts when they say: "you're not sleeping, ma'am, we'll give you a little pill". It's quicker and more effective (Patient).

There are some who are quite OK with medication, that's just what they like, to have a quick solution (Social Worker).

I prescribe them Ativan. I know there are other things we could do, but we don't have enough time, ok, I honestly admit it, I go with the easy solution (Hematologist-oncologist).

Of course, when I have a patient in front of me who is having trouble sleeping and is on Decadron, I'll often call their doctor to get a prescription for something that will help them sleep because that's where the problem is. If it's somebody... who isn't necessarily on Decadron and the situation isn't urgent, long term problems, well, that's different...maybe I won't look for an immediate prescription, sometimes I'll get in touch with their family doctor (Pharmacist).

My initial reaction would be to say that unfortunately patients stay too long on their medication... then, well, they get used to it, they'll have trouble stopping and that's not because they don't want to (Administrator).

\section{Mapping of Barriers and Facilitators using the CFIR Framework}

Barriers and facilitators to implementing a stepped care CBT-I in cancer clinics were grouped into larger domains based on the CFIR model: 1) characteristics of individuals; 2 ) intervention characteristics, 3 ) inner setting; and 4) process ((22), https://cfirguide.org).

\section{Characteristics of Individuals}

Knowledge and beliefs about CBT-I. CBT-I is an effective treatment and an additional tool to offer to cancer patients who have difficulty sleeping, but it does not appear to be very well known by cancer care providers in general and remains underutilized. 
I think maybe it's more a combination of the two, medication combined with a therapy, which in my view, should be the best (Social Worker).

I think it's not yet as well-known as one would like, psychotherapy for sleep works very well, in that regard there is still a lot of work to make people aware of it (Psychologist).

Comorbidity. Participants, particularly psychologists, stated that it might be a challenge to take into account the common comorbidity of insomnia with other psychological disorders and pain.

Insomnia and sleep disturbances don't just present by themselves (Psychologist).

It's a very structured treatment. In real life insomnia or sleep disturbances don't just present by themselves... you need to be very flexible in terms of how you intervene (Psychologist).

Motivation. Some cancer care providers discussed the degree of motivation participants would need to complete and adhere to a stepped care CBT-I beginning with an Internet-based (entirely self-administered) CBT-I. The intervention requires significant lifestyle changes and one must be prepared to make the effort to put the strategies into practice.

It will depend on the people, on how motivated they are... the intensity of their insomnia, I think, will also influence their motivation (Social Worker).

Sure it's a bit harder to take the trouble of going on the Internet and doing the modules every week, for six weeks than to say I'm going to see a psychologist every week for six weeks. It takes more discipline (Nurse).

But you have to be ready to want to do that. You have to go when you feel like it. If you go but you don't really want, that isn't pleasant. You have to pick the right moment when you most feel like it (Patient).

Preferences. The importance of taking patients' preferences into account was also mentioned as an important factor by the health care providers. For instance, patients who want a rapid solution to their problem would probably be better off if offered a pharmacological intervention.

Of course, you have to respect peoples' preferences, some will want the quickest solution and they also won't have the energy to invest in therapy (Psychologist).

\section{Intervention Characteristics}

Accessibility. On one hand, the lack of knowledge and access to the Internet was reported as a critical barrier for a stepped care CBT-I whose first step is an internet-based intervention. This barrier is likely to affect the involvement of patients living in remote areas, older people, and those with a low literacy (including health literacy) and lower socioeconomic status. This should be taken into account when thinking about referring the patient to the program. 
You'll also need to target a certain clientele, because there are patients who aren't on the Internet or who are illiterate or who can't read, who don't know how computers work (Nurse).

I think there's a certain segment of our clientele who are quite elderly...they're likely to feel less comfortable or attracted by that (Social Worker).

Maybe not everybody is able to access it and has the knowledge (Patient).

On the other hand, the proposed intervention was perceived as highly accessible in the sense that it is simple to use and easy to understand, while being less costly for the organization. Moreover, the possibility to complete the treatment program at home, at a time and pace convenient for the patients, was identified as one of the clear advantages of the web-based CBT-I. Also, some patients who already have several medical appointments may refuse a psychological intervention because of transportations issues and some may have less energy to engage in a face-to-face intervention.

Personally, I found it very accessible. For anybody, it's simple and easy to understand (Patient).

The clientele it reaches, access is easy, once it's up and running, it no longer costs anything (Social Worker).

Even for somebody undergoing treatment, they can take it in small steps. They can watch it whenever they want, watch it again if they want (Patient).

The advantage is that the patient won't have to go anywhere. Already there are some... who are very reluctant to travel, they already have a lot of appointments...(Nurse Navigator).

Short and long-term beneficial effects and impact on quality of life. Participants, especially patients and psychologists, emphasized the greater efficacy of CBT-I over pharmacotherapy and praised the long-term effects of CBT-I and its overall beneficial effect on quality of life.

In any case I find it more effective. As someone who has already taken anxiolytics, personally, I felt numb the next morning (Patient).

Psychological therapy is a lot better. Sure it's longer, but it lasts over time and the strategies remain (Patient).

It's just as effective as medication if you take the two by themselves... for example, the great thing about therapy, of course, for anyone who is willing to commit themselves to it, because doing therapy is, in fact, demanding, is that the gains remain over the long term, so medication might serve as a band aid, but as soon as you stop, the bad habits are still there, with therapy the changes are more sustainable over time (Psychologist).

We know that sleep has an impact on the quality of life, mood, anxiety and a lot of things (Psychologist). 


\section{Inner Setting}

Time and resources. On one hand, the lack of time and resources, both human and material, emerged as a major possible barrier to implementation. Stakeholders expressed their concern that the implementation of CBT-I in their clinical setting would increase the overload that they were already experiencing. Some also mentioned that the additional costs for hospitals could be another barrier.

Somehow staff will need enough time to do it, in other words, there should be enough resources (Administrator).

On the part of the nurses, when they talk to patients, they need to explain it, sell it, but that takes time (Technologist).

The thing is, the staff in hospitals already seem to me to be up to their necks in work, so it's like saying: give them training, explain once again that insomnia isn't a false problem... my own perception of the healthcare system is that it's clogged (Patient).

Considering the current lack of psychologists and that they all look overworked. Maybe they'll also have difficulties. (Nurse).

As a psychologist who may end up with these patients, if we allow self-referrals, it'll be chaos (Psychologist).

On the other hand, several stakeholders mentioned that the program may promote patients' autonomy, could reduce the strain on resources and would fit well into routine care. Some also said that, while initially it might be perceived as contributing to their overload, the stepped care CBT-I could actually reduce it, at least for some providers, by providing a new service for an unmet need.

For us it might in some way reduce our workload because these people call us for these kinds of problems. The fact is it might already address the issue before it occurs (Nurse Navigator).

At first it might look a little like an extra burden, but after if it's something that can help (Nurse Navigator). Inside the treatment room, it's when we see the patient's set of side effects, it isn't something you can easily find resources for (Nurse).

Maybe we'll save time. Maybe there will be fewer demands on doctors, less strain on the pharmacy, maybe it will save time for other care providers (Technologist).

Remember that there is still many who won't even need the booster sessions, the online modules will be enough for them (Psychologist).

Resistance to change. Resistance to change among cancer care providers was identified as an important potential barrier. Although inevitable, resistance to change was seen as surmountable. Changes are 
necessary to better address patients' needs.

Whenever you have people involved, whether it be a department or young people or middle aged people or the elderly, there will always be resistance, so you just have to deal with it (Technologist).

Every kind of change brings [new] things. One is aware of that, probably it will work out just fine, everything will run smoothly. It's a change and it's a bonus for the patients (Nurse).

\section{Process}

Training. Cancer care providers mentioned that providing a brief training to all types of clinicians eventually involved would be a facilitator to its implementation. Ideally, cancer care providers should have the opportunity to become familiar with the website, the treatment content and the informational documents that will be distributed to patients beforehand.

It's about making adequate training as brief as possible, covering the most important points. Yeah, then the information should be shared with everybody (Administrator).

What's most important in terms of access is to make sure that the people who might be referring are really familiar with the platform (Social Worker).

There really has to be a meeting and they have to explain well, that's how you bring it in and that's the document that you give and we have to read that document ourselves before we hand it [to the patients] so that we can provide a minimum explanation (Technologist).

I think it's a good tool, easy to use, if the nurses in the treatment room have been trained enough to know the contents so they can try to convince the patients to go there [on the website] (Nurse).

They [the booster sessions] are aimed at the needs that remain... for each patient. It's in relation to what the patients have, the kind of problem they still have. So, that means that the professional who sees them has obviously been informed about CBT-I, has a clear idea what it is. (Psychologist).

Engaging. To be successful the implementation should also engage and rely on every professional working in oncology.

In addition, it shouldn't just fall on the shoulders of two or three people but, but on everybody working in oncology (Administrator).

I think that for any kind of innovation, the people involved have to be participants in the process (Psychologist).

Motivation and commitment. The majority of health care providers interviewed expressed a strong desire to commit themselves to the implementation process and to propose the program to their patients. 
My impression is that I would even prescribe it before medication, I wouldn't even do both at the same time, I would have them begin with it and then see how it interacts (Technologist).

Personally, I'm sure that if they explain it to us, how to try to sell it, I'm sure I would be onboard 100\% (Technologist).

Publicity. Participants also mentioned that, in order for the program to be sustainable, that the implementation team should generate a lot of publicity, not only at the beginning, but throughout the whole implementation process.

I think that it will need a lot of, in quotation marks, publicity... we'll have to make sure everyone is on the same page (Hematologist-oncologist).

That goes for everything, whether it's community resources, our services, they'll publicize it all the time, all the time (Social Worker).

\section{Discussion}

The goal of this qualitative study was to collect the information needed to best prepare the implementation of a stepped care CBT-I in the context of cancer. Overall, both hospital employees and patients emphasized the need for better screening and treating cancer-related insomnia. They also unanimously expressed positive comments about CBT-I and the proposed stepped care CBT-I. They identified a few barriers and facilitators to its implementation and the general conclusion was that it was feasible provided certain conditions are met.

About the assessment and management of sleep difficulties, study participants agreed that the current practice was suboptimal. Some clinicians stated that they try to assess sleep more thoroughly when patients disclose having difficulties sleeping (e.g., frequency of difficulties). However, patients are rarely offered CBT-I because of a lack of knowledge of this therapeutic option by a majority of cancer care providers or because psychologists are overbooked ${ }^{1}$. Also, they tend not to refer patients to psychologists when the only complaint is insomnia. At best, pharmacotherapy and sleep hygiene practices are prescribed. Yet, their limitations, especially those of hypnotic medications, were acknowledged (e.g., side effects, lack of sustained effect over time). Accordingly, CBT-I was identified both by clinicians and patients as being the treatment of choice because of its long-term effects and beneficial impact on overall quality of life. The possibility of combining pharmacotherapy and CBT-I for some patients was also raised. Interestingly, patients verbalized that they had not been informed about the possibility that they might develop sleep difficulties during their cancer care trajectory, nor was the presence of such symptoms assessed by any type of first-line cancer care providers. They also mentioned that insomnia and the program should be discussed with patients as early as possible in the cancer care trajectory. These findings duplicate those of a previous qualitative study conducted in cancer patients with sleep difficulties (10). 
Individual barriers were identified by patients for the implementation of the stepped care CBT-I. They mentioned that several health care professionals don't know about CBT-I being the treatment of choice and that information about its nature and efficacy should be provided first. When questioned about the relevance of the stepped care approach involving a web-based intervention as the first step, some hospital employees and patients wondered if all patients would have the necessary motivation to complete this treatment on their own. They also emphasized the importance of taking into account patients' preferences with regard to treatment modality. Some participants, especially psychologists, said that the presence of comorbidity (e.g., depressive or anxiety disorder) could make referral to the program less relevant. Hence, it will be critical to develop clear algorithms to help health professionals decide who to refer to the stepped care CBT-I program.

Barriers related to the intervention included limited access to the Internet for some patients (e.g., living in rural areas with no high-speed connection, no computer or other electronic device at home, lack of computer skills), as well as older age and lower socio-economic status and literacy. Such barriers to Internet-based interventions were also found in other populations (26),(27), which tend to decrease over time given the continued increasing rates of people having access to the Internet and owning a smart phone, a tablet or a computer (28). On the other hand, many advantages of a web-based treatment were also recognized both by hospital professionals and patients, including its lower cost and greater accessibility, as well as the possibility of completing it at home, without involving additional visits to the clinic, at a time convenient for the patient. Taken together, these findings suggest that it is crucial to provide both technical and clinical support in order to better accompany patients completing the webbased CBT-I.

As expected, the lack of time and human resources were the external (inner setting) barriers that were the most prominently emphasized by administrators, clinicians and even patients. Cancer clinics are already overloaded and the concern was that the program would add to clinicians' burden. Although the first step of the program is entirely self-administered, it still needs to be at least minimally explained when recommended to patients by the health professional. Psychologists working in oncology are also overburdened and the possibility was discussed that there were not enough of them to offer the booster sessions to patients who were still symptomatic after the completion of the web-based phase. Conversely, it was mentioned that the stepped care CBT-I could actually reduce the workload of many types of providers (e.g., oncologists and pharmacists who prescribe hypnotic medications) and could reduce the overall burden on the staff. This would be achieved by providing a new service for an unmet need and for a problem that may manifest otherwise or develop into a more severe problem (e.g., depressive disorder) thus prompting extra phone calls and consultations. As in other implementation studies (23),(29),(30), resistance to change was identified as a possible barrier. Although seen as inevitable, it was also perceived as a temporary issue until everyone understands the advantages of implementing this program in their service. Altogether, the analysis of external barriers reveals that it is crucial to provide information to all stakeholders on the possible consequences of chronic insomnia when left untreated (e.g., increased risk for psychological disorders that are more costly in terms of money and resources needed to treat) and to track early in the process the impact of the implementation 
of stepped care CBT-I on available resources. Based on our previous RCT (17), it is expected that most patients eligible to receive booster sessions will need only one to go into a remission but this needs to be confirmed in the real-world.

Finally, regarding the implementation process modalities, participants expressed the need to offer a prior training to all categories of providers involved in implementation, at a level adjusted to their role. While an extensive training on CBT-I will be required for psychologists offering booster sessions, a much briefer and basic training will be needed for referring providers focusing on how the web-based intervention works and on the content of the intervention in order to be able to "sell it" to their patients. They also emphasized the need to continuously publicize the program all over the hospital both among patients and health professionals.

This study had many strengths. First, focus groups were composed of all relevant stakeholders involved in cancer care for each study site, including administrators. Also, discussions were led by an independent facilitator from a survey firm, which reduces the possibility of a social desirability bias. In addition, the qualitative analysis was conducted by an experienced rater, who was also independent of our research team, thus reducing the potential for experimenter bias. The study also has some limitations as well. Because of recruitment difficulties, the patients' subgroup was predominantly composed of individuals who had received the stepped care CBT-I as part of our previous study (17). While these patients were in the best position to give comments on the intervention and its effects, they were also more likely to be biased positively. Also, participants were in the vast majority women, thus restricting the generalization of findings.

${ }^{1}$ Since 2012, psychotherapy is an act that is reserved to psychologists and other health care professionals having a permit to practice psychotherapy (e.g., primary care physician) in the province of Quebec. CBT-I is a form of psychotherapy according to the definition used by the Office des professions du Québec ("A psychological treatment for a mental disorder, behavioral disturbance or other problem resulting in psychological suffering or distress, and whose purpose is to foster significant changes in the client's cognitive, emotional or behavioral functioning, his interpersonal relations, his personality or his health").

\section{Conclusion}

This qualitative study, which is the first phase of a broader implementation study, provides the necessary insights to prepare the implementation of stepped care CBT-I in cancer clinics. The study also made it possible to engage the main stakeholders who will be involved in this process, thus maximizing its success. Cancer-related insomnia too often remains undetected and undertreated in routine care. CBT-I is recognized as the treatment of choice for insomnia and a successful implementation of a stepped care approach to offer this intervention will contribute to reducing the individual and social burden of cancerrelated insomnia and to increasing patients' overall quality of life. 


\section{Abbreviations}

- CBT-l: cognitive-behavioral therapy for insomnia

\section{Declarations}

\section{Ethics approval.}

This study was approved by the ethics' committee of each participating institution (CHU de QuébecUniversité Laval: \# MP-20-2020-4796; Hôtel-Dieu de Lévis and CISSS Chaudière-Appalaches: \# MEO-202020-711; Institut universitaire en cardiologie et pneumologique de Québec : \#MEO-20-2021-3408).

\section{Consent for publication.}

Not applicable.

\section{Availability of data and materials.}

Not applicable.

\section{Competing interests.}

Josée Savard reports the following conflict of interest: Astellas (consultant). Charles Morin reports the following conflicts of interests: Advisory boards: Eisai, Merck, Sunovion, Pear Therapeutics, Weight Watchers; Research Grant: Idorsia, Canopy Health. The other authors report no conflicts of interest.

\section{Funding.}

This research was funded by the Canadian Institutes of Health Research (\#156376).

\section{Authors' contribution.}

JS : conceptualization, formal analysis, funding acquisition, investigation, methodology, project administration, resources, supervision, writing; CF: formal analysis, investigation, project administration, writing; MPG: conceptualization, formal analysis, funding acquisition, investigation, methodology, supervision, writing; ACG: conceptualization, funding acquisition, methodology, resources; LB: conceptualization, funding acquisition, methodology, writing; CMM: conceptualization, funding acquisition, methodology, writing. All authors read and approved the final manuscript.

\section{Acknowledgments.}

The authors would like to sincerely thank all study participants for their time and cooperation in this research.

\section{References}


1. Savard J, Ivers H, Villa J, Caplette-Gingras A, Morin CM. Natural course of insomnia comorbid with cancer: An 18-month longitudinal study. Journal of Clinical Oncology. 2011;29(26):3580-6.

2. Savard J, Savard MH. Insomnia and cancer: Prevalence, nature, and nonpharmacologic treatment. Sleep Medicine Clinics. 2013;8(3):373-87.

3. Daley M, Morin CM, LeBlanc M, Gregoire JP, Savard J. The economic burden of insomnia: Direct and indirect costs for individuals with insomnia syndrome, insomnia symptoms, and good sleepers. Sleep. 2009;32(1):55-64.

4. National Institutes of Health. National Institutes of Health State-of-the-Science Conference Statement on Manifestations and Management of Chronic Insomnia in Adults, June 13-15, 2005. Sleep. 2005;28(9):1049-57.

5. Qaseem A, Kansagara D, Forciea MA, Cooke M, Denberg TD, Clinical Guidelines Committee of the American College of P. Management of chronic insomnia disorder in adults: A clinical practice guideline from the American College of Physicians. Annals of Internal Medicine. 2016;165(2):125-33.

6. Howell D, Oliver TK, Keller-Olaman S, Davidson J, Garland S, Samuels C, et al. A pan-canadian practice guideline: Prevention, screening, assessment, and treatment of sleep disturbances in adults with cancer. Supportive Care in Cancer. 2013;21(10):2695-706.

7. Espie CA, Fleming L, Cassidy J, Samuel L, Taylor LM, White CA, et al. Randomized controlled clinical effectiveness trial of cognitive behavior therapy compared with treatment as usual for persistent insomnia in patients with cancer. Journal of Clinical Oncology. 2008;26(28):4651-8.

8. Garland SN, Carlson LE, Stephens AJ, Antle MC, Samuels C, Campbell TS. Mindfulness-based stress reduction compared with cognitive behavioral therapy for the treatment of insomnia comorbid with cancer: A randomized, partially blinded, noninferiority trial. Journal of Clinical Oncology. 2014;32(5):449-57.

9. Savard J, Simard S, Ivers H, Morin CM. Randomized study on the efficacy of cognitive-behavioral therapy for insomnia secondary to breast cancer: II - Immunological effects. Journal of Clinical Oncology. 2005;23(25):6097-105.

10. Davidson JR, Feldman-Stewart D, Brennenstuhl S, Ram S. How to provide insomnia interventions to people with cancer: Insights from patients. Psycho-Oncology. 2007;16(11):1028-38.

11. Koffel EA, Koffel JB, Gehrman PR. A meta-analysis of group cognitive behavioral therapy for insomnia. Sleep Medicine Reviews. 2015;19C:6-16.

12. Savard J, Ouellet M-C. Handbook of Sleep Disorders in Medical Conditions: Academic Press; 2019.

13. van Straten A, Cuijpers P. Self-help therapy for insomnia: A meta-analysis. Sleep Medicine Reviews. 2009;13(1):61-71.

14. Ho FY-Y, Chung K-F, Yeung W-F, Ng TH, Kwan K-S, Yung K-P, et al. Self-help cognitive-behavioral therapy for insomnia: a meta-analysis of randomized controlled trials. Sleep medicine reviews. 2015;19:17-28.

15. Zachariae R, Lyby MS, Ritterband LM, O'Toole MS. Efficacy of internet-delivered cognitive-behavioral therapy for insomnia-a systematic review and meta-analysis of randomized controlled trials. Sleep 
medicine reviews. 2016;30:1-10.

16. Savard J, Ivers H, Savard MH, Morin CM. Is a video-based cognitive-behavioral therapy for insomnia as efficacious as a professionally-administered treatment in breast cancer? Results of a randomized controlled trial. Sleep. 2014;37(8):1305-14.

17. Savard J, Ivers H, Savard M, Morin CM, Caplette-Gingras A, Bouchard S, et al. Efficacy of a stepped care approach to deliver cognitive behavioral therapy for insomnia in cancer patients: A noninferiority randomized controlled trial. . Submitted.

18. Espie CA. "Stepped Care": A health technology solution for delivering cognitive behavioral therapy as a first line insomnia treatment. Sleep. 2009;32(12):1549-58.

19. Mack LJ, Rybarczyk BD. Behavioral treatment of insomnia: A proposal for a stepped-care approach to promote public health. Nature and Science of Sleep. 2011;3:87-99.

20. Baglioni C, Altena E, Bjorvatn B, Blom K, Bothelius K, Devoto A, et al. The European Academy for Cognitive Behavioural Therapy for Insomnia: An initiative of the European Insomnia Network to promote implementation and dissemination of treatment. Journal of sleep research. 2020;29(2):e12967.

21. Grimshaw J, Eccles M, Tetroe J. Implementing clinical guidelines: current evidence and future implications. Journal of Continuing Education in the Health Professions. 2004;24(S1):S31-S7.

22. Damschroder LJ, Aron DC, Keith RE, Kirsh SR, Alexander JA, Lowery JC. Fostering implementation of health services research findings into practice: a consolidated framework for advancing implementation science. Implementation science. 2009;4(1):1-15.

23. Geerligs L, Rankin NM, Shepherd HL, Butow P. Hospital-based interventions: a systematic review of staff-reported barriers and facilitators to implementation processes. Implementation Science. 2018;13(1):36.

24. Inc. QI. NVivo 11.0 Qualitative Data Analysis. 2019.

25. Fereday J, Muir-Cochrane E. Demonstrating rigor using thematic analysis: A hybrid approach of inductive and deductive coding and theme development. International Journal of Qualitative Methods. 2006;5(1):80-92.

26. Ariens LF, Schussler-Raymakers FM, Frima C, Flinterman A, Hamminga E, Arents BW, et al. Barriers and facilitators to eHealth use in daily practice: perspectives of patients and professionals in dermatology. Journal of medical Internet research. 2017;19(9):e300.

27. Musiat P, Goldstone P, Tarrier N. Understanding the acceptability of e-mental health-attitudes and expectations towards computerised self-help treatments for mental health problems. BMC psychiatry. 2014;14(1):109.

28. Canada S. Canadian Internet Use Survey. 2019.

29. Kooij L, Groen WG, van Harten WH. Barriers and Facilitators Affecting Patient Portal Implementation from an Organizational Perspective: Qualitative Study. Journal of medical Internet research. 2018;20(5):e183. 
30. Stone AB, Yuan CT, Rosen MA, Grant MC, Benishek LE, Hanahan E, et al. Barriers to and facilitators of implementing enhanced recovery pathways using an implementation framework: a systematic review. JAMA surgery. 2018;153(3):270-9.

\section{Table}


Table 1

Composition of focus groups for each participating hospital.

\begin{tabular}{|c|c|c|c|}
\hline Hospital & Occupation & No. & Sex \\
\hline Hôtel-Dieu de Lévis & Administrator & $1^{1}$ & Male $=1$ \\
\hline \multirow[t]{6}{*}{$(n=8)$} & Nurse & 1 & Females $=7$ \\
\hline & Nurse navigator & 1 & \\
\hline & Physician (radio-oncology) & 1 & \\
\hline & Psychologist & 1 & \\
\hline & Social worker & 1 & \\
\hline & Technologist & 2 & \\
\hline \multirow[t]{3}{*}{ CISSS de Chaudière-Appalaches $(n=4)$} & Administrator & $1^{1}$ & Male $=0$ \\
\hline & Nurse clinician & 2 & Females $=4$ \\
\hline & Nurse navigator & 1 & \\
\hline \multirow{7}{*}{$\begin{array}{l}\text { L'Hôtel-Dieu de Québec } \\
(n=13)\end{array}$} & Administrator & 2 & Male $=0$ \\
\hline & Nurse & 1 & Females $=13$ \\
\hline & Nurse navigator & 2 & \\
\hline & Physician (radio-oncology) & 1 & \\
\hline & Social worker & 1 & \\
\hline & Psychologist & 5 & \\
\hline & Technologist & 1 & \\
\hline \multirow[t]{5}{*}{ Hôpital du St-Sacrement $(n=9)$} & Nurse navigator & 2 & Male $=2$ \\
\hline & Pharmacist & 1 & Females $=7$ \\
\hline & $\begin{array}{l}\text { Physician (hematology- } \\
\text { oncology) }\end{array}$ & 1 & \\
\hline & Psychologist & 1 & \\
\hline & Social worker & $\checkmark$ & \\
\hline
\end{tabular}

${ }^{1}$ One administrator was working in two hospitals, hence she took part to two focus groups. 


\begin{tabular}{|lllll|}
\hline Hospital & Occupation & No. & Sex \\
\hline Hôpital de l'Enfant-Jésus $(n=7)$ & Head of hematology- & 1 & Male $=1$ \\
& oncology department & 2 & Females = 6 \\
& Nurse navigator & 3 & \\
& Psychologist & 1 & \\
& Social worker & & \\
\hline $\begin{array}{l}\text { Institut universitaire de cardiologie et de } \\
\text { pneumologie de Québec }(n=9)\end{array}$ & Nurse & 5 & Females $=9$ \\
& Nurse navigator & 1 & \\
& Administrator & 1 & \\
& Physician (pneumology) & 1 & \\
& Psychologist & 1 & \\
\hline${ }^{1}$ One administrator was working in two hospitals, hence she took part to two focus groups. \\
\hline
\end{tabular}

\section{Supplementary Files}

This is a list of supplementary files associated with this preprint. Click to download.

- SRQRGuidelinesChecklist.doc 